\title{
A 2D Fourier tool for the analysis of photo-elastic effect in large granular assemblies
}

\author{
Danuta Leśniewska* \\ Koszalin University of Technology, Department of Civil, Geodetic and Environmental Science, 74-453 Koszalin, Poland
}

\begin{abstract}
Fourier transforms are the basic tool in constructing different types of image filters, mainly those reducing optical noise. Some DIC or PIV software also uses frequency space to obtain displacement fields from a series of digital images of a deforming body. The paper presents series of 2D Fourier transforms of photo-elastic transmission images, representing large pseudo 2D granular assembly, deforming under varying boundary conditions. The images related to different scales were acquired using the same image resolution, but taken at different distance from the sample. Fourier transforms of images, representing different stages of deformation, reveal characteristic features at the three ('macro-', 'meso-' and 'micro-') scales, which can serve as a data to study internal order-disorder transition within granular materials.
\end{abstract}

\section{Introduction}

Fourier transforms are the basic tool in constructing different types of image filters, mainly those reducing optical noise. Some DIC or PIV software also uses frequency space to obtain displacement fields from a series of digital images of a deforming body. There exists one more possible way of utilizing 2D Fourier transforms for granular materials - they can be regarded as a direct subject of analysis themselves [1], similarly to Laue patterns in crystallography (physical Fourier transforms). The Lauegrams are used to measure internal structure of crystals from distances between some ordered intensity peaks. The idea of the approach proposed in this paper comes from the certain similarities between poly-crystals and granular materials - the former also show some kind of structural order, especially if so called 'force chain' network is studied. Investigation of 'force chains' structure in granular materials started about 1920 and was continued by several researchers up to now - the lack of space does not allow to discuss their achievements here.

The important problem still left to be solved is the quantitative interpretation of the structure of 'force chain' network, observed in photo-elastic experiments. Some objective tool is necessary to measure characteristic distances and angles od these networks and to connect them with the way granular material deforms. The additional problem is related to scale dependence - the force chain network seem to be the macro-scale feature, but it is necessary to determine some physical limits of the phenomenon. To illustrate the problem, the paper presents selected series of 2D Fourier transforms of photo-elastic transmission images, representing large (consisting of $\sim 1 \mathrm{e}+6$ grains) granular assembly, acquired during small scale geotechnical model tests.

\section{Granular assembly and test details}

The assembly investigated in this work consisted of small, uniformly graded glass granules (mass median diameter Dso 1 $1 \mathrm{~mm},[2,3]$ ) and can be classified as pseudo 2D, because it is placed in a narrow rectangular box $(30 \mathrm{~cm} \mathrm{x}$ $27 \mathrm{~cm} \times 2 \mathrm{~cm}$ ), enforcing plane strain condition on macro (sample) scale.

It has to be remembered that individual grains are spherical and form several layers, none of them perfectly plane. The detailed description of the test layout was published elsewhere $[2,3]$.

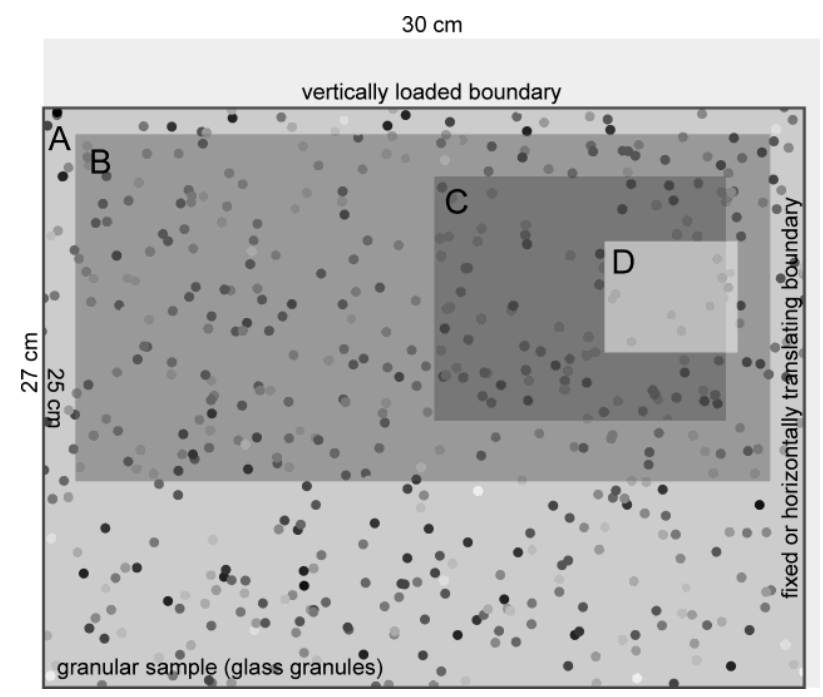

Fig. 1. The schematic view of the areas of granular sample: $\mathrm{A}-$ view of the whole sample, $\mathrm{C}$ - first close-up, D - second closeup. $\mathrm{B}-$ part of the area A, used in the analysis.

\footnotetext{
* Corresponding author: danuta.lesniewska@tu.koszalin.pl
} 
The granular assembly deforms under varying boundary conditions (top boundary is loaded vertically and right boundary translates horizontally, Fig.1). Principle of integrated photo-elasticity is used (transparent grains immerged in immersion liquid - clove oil here). Specific type of circular polariscope was employed, equipped with rectangular polariser and analyser and thus giving much better quality of photo-elastic images of granular sample under load, than standard polariscopes.

The photographs presented in Fig.2, column A, were taken using RGB scientific grade camera and later transformed to grayscale images to calculate Fourier transforms. Fourier transforms of images, representing different stages of real sample deformation (Fig.2, column B) and reverse Fourier transforms of artificial images (Fig.3, column B) were calculated using commercial DaVis8 software by LaVision.

Separate images of three different areas of the sample (B, C and D, Fig.1) were analysed and are presented in this paper. They represent:

(1) the whole width of the sample ('macro' scale, the area of an image equal to $\sim 250 \times 200 \mathrm{D}_{50}, \mathrm{~B}$, $\sim 28 \mathrm{~cm} \times 15 \mathrm{~cm}$, Fig. 1 ),

(2) medium size part of the sample ('meso-scale', the area of an image equal to $\sim 100 \times 80 \mathrm{D}_{50}, \mathrm{C}$, $\sim 10 \mathrm{~cm} \times 8 \mathrm{~cm}$, Fig. 1 ),

(3) (3) small part of the sample, representing the interior of the expected shear band ('microscale', the area of an image equal to $\sim 40 \times 30$ D $50, \sim 5 \mathrm{~cm} \times 4 \mathrm{~cm}$, Fig. 1 ).

The area B was selected to be analysed instead of the whole sample view A, to exclude any possible influence of some optical imperfectness found in the lower part of the images, on the shape of Fourier transforms.

The imperfection was created by slightly different colouring of the immersion liquid used in the tests. The images related to different scales were acquired using the same image resolution, but were taken at different distances from the granular sample. As a result the average grain is represented by $\sim 10 \times 10$ pixels in the areas $\mathrm{A}$ and $\mathrm{B}, \sim 25 \times 25$ pixels in the area $\mathrm{C}$ and $\sim 50 \times 50$ pixels in the area $\mathrm{D}$ (Fig.1). The last resolution gives the opportunity to discern the edges of individual grains and to study the sample at micro scale.

\section{Discussion of Fourier transforms}

Fig.2, column A, shows different areas of the granular sample, photographed in circularly polarised light, using full camera resolution $(\sim 2400 \times 2000$ pixels $)$. All images show photo-elastic response of the granular sample averaged over the sample thickness (about 20 layers of grains).

\subsection{Real images}

Fig.2 (column A) represents some typical experimental data - photo-elastic response of $2 \mathrm{~cm}$ thick granular sample, consisted of uniformly graded glass granules.
Due to dimensional uniformity of grains some crystallization effect can be expected.

The granular sample undergoes growing external loading on the top boundary (Fig.2, column A, rows 1-2, 6-7), some unloading (Fig.2, column A, rows 3 and 6) and some uniform displacement of the right boundary (Fig.2, column A, rows 3, 5, 7). Development of complex 'force chain' system, demonstrating clear geometrical structure can be seen in all cases, depending mainly on the value of external load. Dark bands appearing at the right side of the images coincide with shear band location, what was checked by PIV (DIC) method, [2,3]. Such dark, arched band is most clearly visible on Fig. 2 column A, row 4 and 5. Darker colour on the image means weaker 'force chains', suggesting that some force chains must have been broken within the shear band.

It is worthy of noting that even after total unloading some strong 'force chains' remain stable at the bottom of the sample (Fig.2, column A, row 6), thus the sample does not fully return to the initial isotropic state.

The order of the 'force chain' system, clearly visible in Fig. 2, column A, row 2 and others, by a naked eye (two privileged directions suggesting induced stress anisotropy) failed to be captured by any standard image analysis tools. Possible reason for that is the fact, that the 'force chains' are numerous and very thin structures (usually much thinner than the average grain diameter) and cannot be turned into continuous structures by any edge finding filter or image segmentation algorithm.

In effort to extract some quantitative information concerning organization of force chain network the photoelastic images were transformed into frequency spectrum - Fig.2, column B shows Fourier transforms of images shown in Fig.2, column A.

It can be observed that Fourier transforms change with the value of external load at 'macro scale' (Fig.2, column A, rows 1-3), but any significant difference exist only between the first phase of relatively low value of external load ( $p=0.2 \mathrm{MPa}$, Fig. 2, column B, row 1) and the higher load levels

There exists the ring-shaped distribution in the power spectrum of Fourier transforms throughout the whole loading range at 'macro scale' - it is well known from crystallography that such a ring demonstrates periodicity at intermediate spatial frequencies, that is equivalent in all directions in the image (in case of this paper this is most probably the manifestation of the sample 'crystallization' due to significant mono dispersity of glass granules). In course of the loading process other objects appear in Fourier power spectrum, most likely related to emerging and stabilizing force chain network.

Fig.2, rows 4-6 represent the 'meso-scale' of the sample. If the Fourier transforms are compared to ones, obtained for 'macro-scale' (Fig.2, rows 1-3), it seems obvious, that the effect of 'crystallization' (ring-shaped distribution) is observable only at bigger scale. Fig.2B (row 6) shows that the sample unloading is equivalent to the transition from more anisotropic to more isotropic state. Fig.2B (row 7) suggests that at the scale close to a shear band width the stress anisotropy is less distinct, than at larger scales. 


\begin{tabular}{|c|c|c|}
\hline $\begin{array}{l}\text { Area and the test } \\
\text { conditions (Fig.1) }\end{array}$ & $\begin{array}{l}\text { A. System of 'force chains' photographed } \\
\text { in circularly polarized light (dark field) }\end{array}$ & $\begin{array}{l}\text { B. Fourier transforms corresponding to images } \\
\text { from column A }\end{array}$ \\
\hline $\begin{array}{l}\text { 1. Area B in Fig. } 1 \\
\text { Vertical surcharge } \\
\text { of the top boundary } \\
\mathbf{p}=\mathbf{0 . 2 M P a} \text {, right } \\
\text { boundary fixed }\end{array}$ & & \\
\hline $\begin{array}{l}\text { 2. Area B in Fig. } 1 \\
\text { Vertical surcharge } \\
\text { of the top boundary } \\
\mathbf{p = 1 . 6 M P a} \text {, right } \\
\text { boundary fixed }\end{array}$ & & \\
\hline $\begin{array}{r}\text { 3. Area B in Fig.1 } \\
\text { Vertical surcharge } \\
\text { of the top boundary } \\
\mathbf{p = 0 . 8 M P a} \\
\text { (unloading), right } \\
\text { boundary } \\
\text { displacement of } \\
\mathbf{d = 1 . 2 5 m m}\end{array}$ & & \\
\hline $\begin{array}{r}\text { 4. Area } \mathbf{C} \text { in Fig. } 1 \\
\text { Vertical surcharge } \\
\text { of the top boundary } \\
\mathbf{p}=\mathbf{1 . 6 M P a} \text { right } \\
\text { boundary initial } \\
\text { displacement } \\
\mathbf{d}=\mathbf{1 . 2 5 m m} \text { - no dis- } \\
\text { placement increment }\end{array}$ & & \\
\hline $\begin{array}{r}\text { 5. Area } \mathbf{C} \text { in Fig. } 1 \\
\text { Vertical surcharge } \\
\text { of the top boundary } \\
\mathbf{p}=\mathbf{0 . 8 M P a} \text {, right } \\
\text { boundary initial } \\
\text { displacement } \\
\mathrm{d}_{0}=\mathbf{1 . 2 5 \mathrm { mm } \text { , added }} \\
\mathbf{d =} \mathbf{3 . 7 5 m m} .\end{array}$ & & \\
\hline $\begin{array}{r}\text { 6. Area } \mathbf{C} \text { in Fig. } 1 \\
\text { Vertical surcharge } \\
\text { of the top model's } \\
\text { boundary } \mathbf{p}=\mathbf{0 M P a} \\
\text { (unloading), right } \\
\text { boundary initial } \\
\text { displacement } \\
\mathrm{d} 0=1.25 \mathrm{~mm} \text {, total } \\
\mathbf{d =}=\mathbf{3 . 7 5 m m} .\end{array}$ & & \\
\hline $\begin{array}{r}\text { 7. Area } \mathbf{D} \text { in Fig. } 1 \\
\text { Vertical surcharge } \\
\text { of the top model's } \\
\text { boundary } \\
\text { p=1.6MPa - } \\
\text { unloading, right } \\
\text { boundary initial } \\
\text { displacement } \\
\text { do }=5 \mathrm{~mm} \text {, added } \\
\mathbf{d = 0 . 0 6 2 5 m m} .\end{array}$ & & \\
\hline
\end{tabular}

Fig. 2. Examples of photo-elastic images taken at different scales (column A) and their Fourier transforms (column B). 


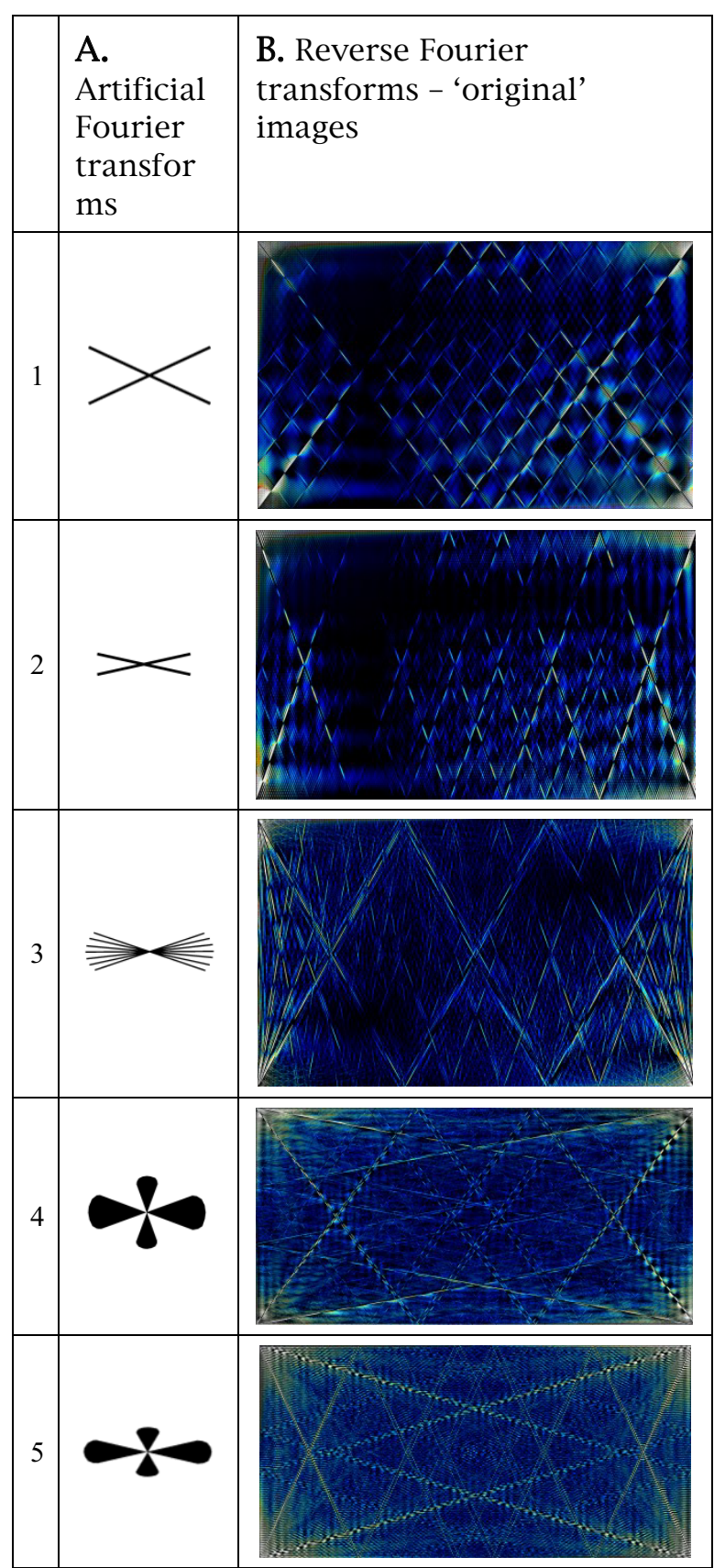

Fig. 3. Idealized components of Fourier transforms (A), sketched on the base of Fig.2, and their reverse (B), showing structures corresponding to different arrangement of the real force chain networks.

\subsection{Artificial images}

As the geometric features visible in Fig.2A (rows 2 and 3) are quite regular, it is possible to recognize the ones directly related to force chains and their arrangement. It can be agreed that Fourier transforms of Fig.2B (rows 2 and 3) consists of a ring-shaped area and a double fan, which can have complicated internal structure, but is most probably related to anisotropic force chain networks, visible in Fig.2, column A. At 'meso scale' Fourier transforms show lack of ring-shaped object and instead the second, smaller, vertically oriented double fun is present (Fig2B, rows 4-6). Column A of Fig.3 contains artificial images of features which could be recognized in Fig.2B (artificial Fourier transforms). Idealised elements of the fun present at macro-scale are postulated in column A, rows 1-3. The reverse of Fourier transforms calculated in Fig.3 (column B, rows 1-3) show that the angle of the fun is responsible for the range of directions of 'force chains' allowed in given experimental conditions - this range is visibly limited. The external directions (the edges of the fun) define the main 'force chain' network (Fig.3B, rows 1 and 2), the inside of the fun is responsible for the set of weaker 'force chains' directions (Fig.3B, row 3). Also some information on changing length of 'force chains' could be deduced from the dimensions of double fan. Two double funs, characteristic for 'meso scale' are approximated as continuous features in Fig.3A, rows 4 and 5. Corresponding reverse Fourier transforms are shown in Fig.3B, rows 4 and 5. Their structure suggests the presence of second set of two privileged directions in the granular sample, more horizontally oriented. Such family of lines exists in real images - they are dark, in contrast to bright 'force chains' and best visible in reality in Fig.2A, row 7 ('micro-scale'), but also present at 'meso scale'. Their exact nature has to be find yet.

\section{Summary and conclusions}

Fourier transforms of 'force chain' systems in granular materials reveal characteristic features at the three scales, which can serve as a data to study internal order-disorder transition within granular materials. Simplified artificial 'Fourier transforms', after reversing them to artificial 'original images', give insight into different components of 'force chains' network, produced by collective photoelastic response of the real assembly. Comparison between artificial and real images at three scales are presented in the paper to the limited extent. Proposal of determining some characteristic material lengths, like average dimensions of 'force chains' mesh, average characteristic distance between neighbouring 'force chains', average length of 'force chains' at different stages of deformation, is under preparation.

\section{Acknowledgement}

This work was done within the frame of the project nr.2011/03/B/ST8/05865, Polish National Science Centre.

\section{References}

1. T. Prum, R.H. Torres, Integr. Comp. Biol. 43, 591602 (2003)

2. D. Lesniewska, D. Muir Wood, J Eng Mech 135:1038-1054 (2009)

3. D. Muir Wood, D. Lesniewska, Granul Matt 13:395415 (2011) 